TECHNICAL NOTE

M. Adachi

H. Kabasawa

E. Kawaguchi

\section{Depiction of the Cranial Nerves Within the Brain Stem with Use of PROPELLER Multishot Diffusion- Weighted Imaging}

\begin{abstract}
SUMMARY: Despite the recent progress of MR imaging, visualization of the cranial nerves within the brain stem has not been accomplished. Periodically rotated overlapping parallel lines with enhanced reconstruction (PROPELLER) multishot diffusion-weighted imaging, an effective method for compensating for motion and distortion, offers high-quality diffusion-weighted images. We succeeded in depicting the cranial nerves within the brain stem in some subjects by using this method with motion-probing gradient applied in the superior-inferior direction.
\end{abstract}

$\mathbf{T}$ here are many disorders of the cranial nerves, including neoplasm, inflammation, and vascular compression. MR imaging with gradient-echo or fast spin-echo sequences with 3D Fourier transformation has recently enabled depiction of the cranial nerves in the cisterns around the brain stem. ${ }^{1-5}$ However, to the best of our knowledge, visualization of these nerves within the brain stem itself has not been accomplished. We succeeded in depicting the cranial nerves within the brain stem in some of the subjects in this preliminary study by using periodically rotated overlapping parallel lines with enhanced reconstruction (PROPELLER) multishot diffusion-weighted imaging. ${ }^{6,7}$ We present diffusion-weighted images that depict the cranial nerves and the rate of their visualization within the brain stem.

\section{Technique}

We enrolled 8 healthy volunteers: 5 men and 3 women; mean age, 33.6 \pm 9.3 years. We explained the purpose of the MR study to the volunteers and obtained their prior consent.

We performed the MR study in all subjects by using a 1.5T superconducting system (Signa Excite; GE Healthcare, Milwaukee, Wis) and an 8-channel neurovascular phased-array coil. We obtained PROPELLER multishot diffusion-weighted imaging sequences by applying motion-probing gradients (MPG) in 3 orthogonal directions for each section and using fast spin-echo imaging. We obtained 3-orthogonal-axis images (superior-inferior [SI], right-left [RL], anterior-posterior [AP]) as well as isotropic diffusion-weighted images that were generated by averaging the images in the 3 axes. We also obtained short- $\tau$ inversion recovery (STIR) images, which provided good contrast between gray and white matter. ${ }^{8}$ The scan range was from the medulla oblongata to the midbrain.

The parameters for fast spin-echo PROPELLER multishot diffusion-weighted imaging were as follows: matrix size, $192 \times 192$; TR, $6000 \mathrm{~ms}$; TE, $130.9 \mathrm{~ms}$; echo-train length, 24; blade number, 32; band width, $62.5 \mathrm{KHz}$; b-factor, 850 seconds $/ \mathrm{mm}^{2}$; NEX, 4; section thickness, $3 \mathrm{~mm}$; section gap, $0.5 \mathrm{~mm}$; FOV, $24 \times 24 \mathrm{~cm}$; 15 section sections; total imaging time, 12 minutes 54 seconds. The parameters for STIR imaging were as follows: matrix size, $512 \times 256$; TR, $5500 \mathrm{~ms}$;

Received July 25, 2007; accepted after revision on September 24.

From the Department of Radiology (M.A., E.K.), Ohshima Clinic, Yamagata, Japan; and Japan Applied Science Laboratory (H.K.), GE Yokogawa Medical Systems, Tokyo, Japan. Please address correspondence to Michito Adachi, Department of Radiology, Ohshima Clinic, 4-1-14 Sakurada Nishi, Yamagata 990-2321, Japan; e-mail: miadchi@beach. ocn.ne.jp

DOl 10.3174/ajnr.A0957
TE, 25.9 ms; TI, $150 \mathrm{~ms}$; NEX, 2; section thickness, 3 mm; section gap, $0.5 \mathrm{~mm}$; 15 section sections; imaging time, 4 minutes 30 seconds.

These images were evaluated at random by 2 observers (M.A. and E.K), who arrived at their conclusions by mutual consent. They focused on linear high-intensity components in the brain stem.

\section{Results}

Diffusion-weighted images with MPG applied in the SI direction depicted 3 (18.8\%) of 16 oculomotor nerves (Fig 1), 11 (68.8\%) trigeminal nerves (Fig 2), and 7 (43.8\%) vestibulocochlear nerves (Fig 3) as high-intensity linear areas. Diffusion-weighted images with MPG applied in the AP direction depicted only 2 (12.5\%) vestibulocochlear nerves; however, on these images with MPG applied in the AP direction, the vestibulocochlear nerves were depicted unclearly in the tegmentum of the pons, which displayed high signal intensity. No cranial nerves were depicted in the brain stem on diffusion-weighted images with MPG applied in the RL direction or on STIR images. In addition, we could not identify any nucleus in the tegmentum of the brain stem on diffusion-weighted or on STIR images.

\section{Discussion}

PROPELLER, developed by Pipe et $\mathrm{al}^{6,7}$, is a radial data sampling method in which $k$-space is composed in blades, including some rectilinear samplings. This is an effective method for compensating for motion and distortion and offers high-quality multishot diffusion-weighted images without distortion or phase artifact.

We attempted to depict cranial nerves within the brain stem using PROPELLER multishot diffusion-weighted imaging. Visualization of the oculomotor, trigeminal, and vestibulocochlear nerves was achieved in some subjects as linear high-signal intensities on PROPELLER with MPG applied in the SI direction.

Nerve fiber bundles parallel to the direction of the MPG are depicted as low intensities, whereas those perpendicular to the direction of the MPG are depicted as high intensities on diffusion-weighted images because of their anisotropic water diffusion. ${ }^{9}$ From an anatomic standpoint, the cranial nerves originate from the tegmentum and run in an antero-oblique direction. The images with MPG applied in the SI direction are the most perpendicular to the cranial nerve fibers within the brain stem, among those with MPG in 3 directions. In contrast, the white matter components surrounding the cranial nerves (the superior cerebellar peduncle, longitudinal pontine fasciculi, medial longitudinal fasciculus, central tegmental tract, and reticular formation) run almost parallel to the SI 

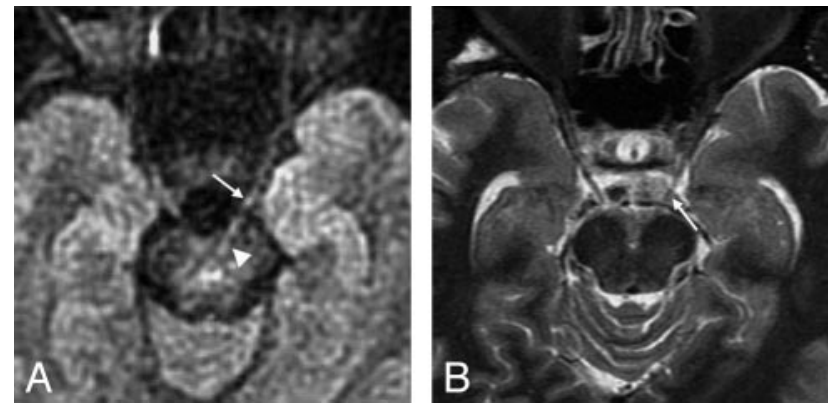

Fig 1. Images of a 44-year-old male volunteer. $A$, Multishot diffusion-weighted image with MPG applied in the SI direction shows the left ophthalmic nerve not only in the cistern (arrow) but also in the midbrain (arrowhead). The bright signals in the center of the midbrain are considered to be the decussation of the superior cerebellar peduncle running in the lateral direction. $B$, STIR image shows the cisternal portion of the left ophthalmic nerve (arrow); however, the nerve is not identified in the midbrain
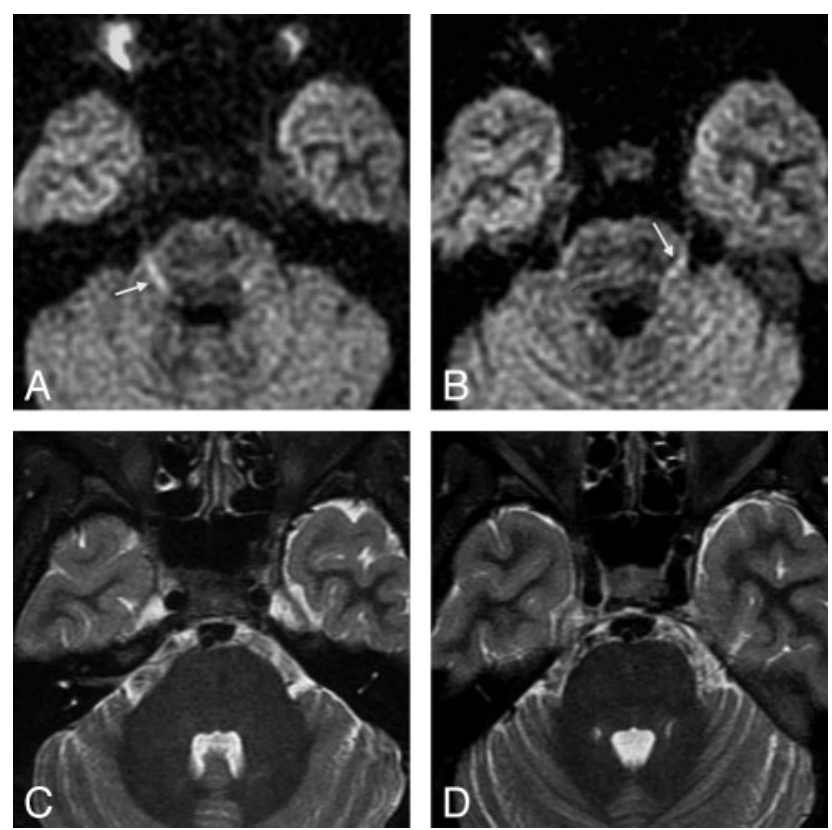

Fig 2. Images of a 30-year-old female volunteer. $A, B$, Multishot diffusion-weighted images with MPG applied in the SI direction show both trigeminal nerves as slightly curved high-intensity lines in the mid-pons (arrows). C,D, The trigeminal nerves are not identified in the mid-pons on the STIR images.
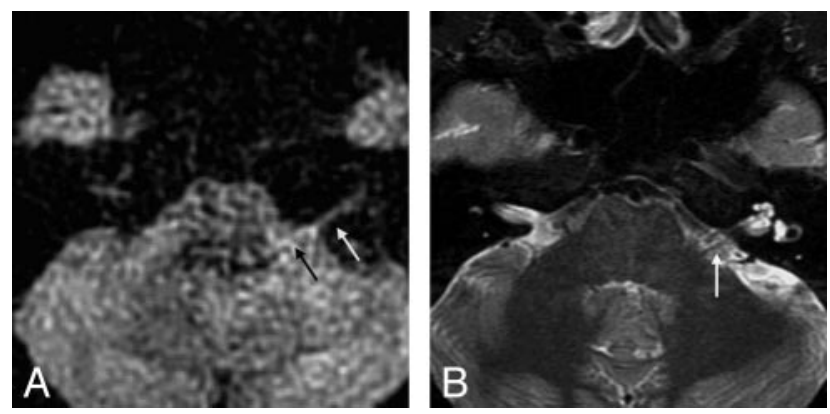

Fig 3. Images of a 34-year-old male volunteer. $A$, Multishot diffusion-weighted image with MPG applied in the SI direction shows the left acoustic nerve as a linear high-intensity line in the brain stem (black arrow) as well as in the cistern (white arrow). B, STIR image shows the left acoustic nerve in the cerebellar-pontine cistern (arrow); however, it is not identified in the brain stem.

direction. The decussation of the superior cerebellar peduncle, the transverse pontine fiber bundles, and the middle cerebellar peduncle then run in a lateral oblique direction. Therefore, diffusion-weighted images with MPG applied in the SI direction should provide good contrast between the cranial nerves and the surrounding white matter.

Numerous recent articles have addressed diffusion tensor imaging and white matter tractography. ${ }^{10-13}$ These reports have established that white matter tractography can reconstruct the major white matter structures, for example, the corticospinal tract. However, no studies have demonstrated the cranial nerves within the brain stem, even with use of the $3 \mathrm{~T}$ superconducting systems that are now available. Therefore, PROPELLER multishot diffusion-weighted imaging is thought to be a valuable tool in revealing anatomic details of the cranial nerves and determining the relationship between the cranial nerves and lesions such as tumors, hematomas, and infarcts.

Although we attempted to depict cranial nerve fibers using 5-to-7-mm-thick images, there was insufficient contrast to discriminate the nerves from the surrounding white matter. We consider that thin-section images, at least $3 \mathrm{~mm}$ thick, would be required to depict cranial nerves. However, the signal-to-noise ratio of 3-mm-thick images is worse than that of 5-to-7-mmthick images, and the quality is currently insufficient to enable evaluation of the cranial nerves in the clinical setting. It would be of great value in future studies to improve the signal-to-noise ratio with use of high-T superconducting system.

\section{Conclusion}

We succeeded in depicting the cranial nerves within the brain stem in some subjects using PROPELLER multishot diffusionweighted imaging with MPG applied in the SI direction. However, the ratio of visualization was insufficient for use in the clinical setting. Improvement in the signal-to-noise ratio remains an issue for future study.

\section{References}

1. Stuckey SL, Harris AJ, Mannolini SM. Detection of acoustic schwannoma: use of constructive interference in the steady state three-dimensional MR. AJNR Am J Neuroradiol 1996;17:1219-25

2. Shibata A, Hosoya T, Kato T, et al. Abducens nerve enhancement in acute ophthalmoparesis. Radiat Med 1998;16:375-77

3. Korogi Y, Nagahiro S, Du C, et al. Evaluation of vascular compression in trigeminal neuralgia by 3D time-of-flight MRA. J Comput Assist Tomogr 1995; 19:879-84

4. Du C, Korogi Y, Nagahiro S, et al. Hemifacial spasm; three-dimensional MR images in the evaluation of neurovascular compression. Radiology 1995;197:227-31

5. Girard N, Poncet M, Caces F, et al. Three-dimensional MRI of hemifacial spasm with surgical correlation. Neuroradiology 1997;39:46-51

6. Pipe JG. Motion correction with PROPELLER MRI: application to head motion and free-breathing cardiac imaging. Magn Reson Med 1999;42:963-69

7. Pipe JG, Farthing VG, Forbes KP. Multishot diffusion-weighted FSE using PROPELLER MRI [published erratum appears in Magn Reson Med 2002;47: 621]. Magn Reson Med 2002;47:42-52

8. Oikawa H, Sasaki M, Tamakawa Y, et al. The substantia nigra in Parkinson disease: proton density-weighted spin-echo and fast short inversion time inversion-recovery MR findings. AJNR Am J Neuroradiol 2002;23:1747-56

9. Moseley ME, Cohen Y, Kucharczyk J, et al. Diffusion-weighted MR imaging of anisotropic water diffusion in cat central nervous system. Radiology 1990;176:439-45

10. Pierpaoli C, Jezzard P, Basser PJ, et al. Diffusion tensor MR imaging of the human brain. Radiology 1996;201:637-48

11. Mori S, Crain BJ, Chacko VP, et al. Three-dimensional tracking of axona projections in the brain by magnetic resonance imaging. Ann Neurol 1999;45:265-69

12. Wakata S, Jiang H, Nagae-Poetscher LM, et al. Fiber tract-based atlas of human white matter anatomy. Radiology 2004;230:77-78

13. Masutani $\mathrm{Y}$, Aoki $\mathrm{S}$, Abe $\mathrm{O}$, et al. MR diffusion tensor imaging: recent advance and new techniques for diffusion tensor visualization. Eur J Radiol 2003;46:53-66 